\title{
Microfinanzas y pobreza rural en México: un análisis con técnicas de propensity score matching*
}

\author{
Jorge Mora-Rivera \\ Instituto Tecnologico y de Estudios Superiores de \\ Monterrey, México \\ jjmora@tec.mx \\ ORCID: http://orcid.org/0000-0003-0838-955I \\ Fernando García-Mora \\ El Colegio de México, A. C., México , México \\ ORCID: http://orcid.org/0000-000I-6858-4952 \\ DOI: https://doi.org/I0.III44/Javeriana.cdrı5-82.mprm \\ Redalyc: http://www.redalyc.org/articulo.oa?id=11757724005
}

Cómo citar este artículo: Mora-Rivera, J., y García-Mora, F. (2018). Microfinanzas y pobreza rural en México: un análisis con técnicas de propensity score matching. Cuadernos de Desarrollo Rural, I5(82), I-19. https://www.doi.org/10.III44/Javeriana.cdri5-82.mprm

Recibido: 29 de marzo de 2018 I Publicación: I8 de diciembre de 2018

\section{Resumen:}

El objetivo de este artículo es estimar el efecto que tiene el uso de las microfinanzas en la pobreza por ingresos de los hogares del sector rural mexicano. Empleando información de la Encuesta Coneval a Hogares Rurales de México 2013 (EnChor 2013) (Consejo Nacional de Evaluación de la Política de Desarrollo Social [Coneval], 2013) y técnicas de propensity score matching, los resultados de esta investigación muestran que el uso de los microcréditos contribuye a disminuir los niveles de pobreza de los hogares rurales en México. Estos hallazgos ponen de manifiesto la necesidad de crear políticas públicas que permitan incorporar a un mayor número de hogares pobres al mercado de las microfinanzas, lo cual incrementaría los niveles de vida de las personas que viven en este sector.

Palabras clave: microfinanzas, pobreza, hogares rurales, matching, México.

\section{Micro-Finances and Rural Poverty in Mexico: An Analysis Based on Propensity Score Matching}

\section{Abstract:}

This article aims to estimate the effect of using the micro-finances on the poverty, measured as the income per Mexican rural household. By 
using the information from the Coneval Survey to Mexican Rural Households 2013 (EnChor 2013) as well as the techniques of Propensity Score Matching, the outcomes from this research show that micro-loans help to reduce the poverty levels among the rural households in Mexico. These findings indicate the need to create public policies that will enable to incorporate higher numbers of poor households into the micro-finance market, which would improve the standard of living of the people in those areas.

Keywords: micro-finances, poverty, rural households, matching, Mexico.

\section{Introducción}

La pobreza es sin lugar a duda uno de los fenómenos que generan mayor preocupación alrededor del mundo (Banerjee y Duflo, 20II; Fosu, 20I7). De acuerdo con estimaciones del Banco Mundial (2017), en 2013, aproximadamente 770 millones de personas (el I0,7\% de la población mundial) vivían con menos de ı,9 dólares al día; la situación se vuelve aún más crítica en el caso de las economías subdesarrolladas, y México no es la excepción. En este país, el panorama de la pobreza es poco alentador, ya que la última estimación oficial de 2016 señala que el $44 \%$ de su población se encontraba ensituaciónde pobreza, cifra que se acentúa más cuando se considera únicamente la condición de los hogares que viven en zonas rurales, pues, para el mismo año, cerca del $60 \%$ de sus habitantes fue catalogado como pobre (Coneval, 2017b).

Dentro de las estrategias en el combate a la pobreza, se ha considerado, a partir de la creación en 1976 del Banco Grameen en Bangladesh, que las microfinanzas son un mecanismo clave para dicho propósito, ya que le proporcionan acceso a los mercados de crédito a un segmento importante de la población pobre que vive en países subdesarrollados (Bateman, 20II; Kasali, Ahmad y Ean, 20I5). Sin embargo, la literatura especializada no ofrece consenso respecto al efecto que el uso de las microfinanzas tiene sobre este fenómeno (Banerjee, Dulfo, Glennerster y Kinnan, 2015; Ghalib, Malki e Imai, 2015; Tarozzi, Desai y Johnson, 2015).

Por un lado, se encuentran estudios que identifican impactos positivos en la reducción de la pobreza originados por las microfinanzas (Attanasio, Augsburg, de Haas, Fitzsimons y Harmgart, 2015; Banerjee et al., 2015; Crepón, Devoto, Dulfo y Pariente, 2015; Ghalib et al., 2015; Imai, Arun y Annim, 2010; Imai y Azam, 20I2; Karlan y Zinman, 2009; Khandker, 2005; Khandker y Samad, 2018; Patel, Patel y Patel, 20I8; Pitt y Khandker, 1998; Sani, Khan, Ahmed y Aziz, 2017; Sultana, Jamal y Najaf, 2017). Por otro lado, existen investigaciones que muestran un impacto nulo o incluso negativo para el mismo propósito (Banerjee y Jackson, 2017; Morduch, I998; Tarozzi et al., 2015). Por lo tanto, y bajo este escenario de evidencias contrapuestas, la presente investigación tiene como propósito calcular el efecto que tiene el uso de las microfinanzas en los niveles de pobreza por ingresos de los hogares del sector rural mexicano y, con ello, contribuir a la discusión académica sobre el tema.

Uno de los desafíos más importantes cuando se analiza el efecto de las microfinanzas en la pobreza es el sesgo por autoselección (Ghalib et al., 2015; Imai et al., 2010), es decir, la decisión de solicitar y usar algún crédito no es aleatoria; de hecho, los hogares que emplean algún préstamo se autoseleccionan dadas ciertas características sociodemográficas y económicas probablemente distintas a las de aquellos que no lo hacen. Para minimizar este problema, y considerando que el grupo que no utiliza crédito debe estar conformado por hogares con características muy similares a los que conforman el grupo que sí lo hace (Caliendo y Kopeinig, 2008), en esta investigación se emplea la metodología conocida como propensity score matching (PSM). Los datos utilizados provienen de la Encuesta Coneval a Hogares Rurales de México 2013 (EnChor 2013) (Coneval, 2013), que permite 
obtener información sobre las características socioeconómicas de los miembros de los hogares, de sus actividades productivas y del acceso a y la participación en los sistemas financieros formales e informales, así como del acceso a la alimentación.

Estudiar los efectos de las microfinanzas en la pobreza es importante por varias razones. En primer lugar, México es uno de los países que a nivel mundial cuenta con más instituciones y programas sociales cuyo propósito es otorgar crédito a familias que se encuentran en situación de pobreza; por lo tanto, resulta indispensable analizar el éxito o fracaso del uso de dichos recursos. Segundo, el número de investigaciones que emplean alguna metodología no descriptiva para analizar la relación entre las microfinanzas y la pobreza en México es realmente escaso. En tercer lugar, para el caso mexicano, este artículo es uno de los primeros en implementar una metodología de efectos de tratamiento para evaluar el impacto del acceso a las microfinanzas en la pobreza por ingresos. Cuarto, analizar este problema es importante ya que permite sugerir medidas de política pública orientadas a promover mecanismos que contribuyan a disminuir la pobreza rural que prevalece en México y en América Latina.

El resto del artículo está organizado de la siguiente manera. La segunda sección contiene una revisión de la bibliografía relevante en el tema; los datos y las estadísticas descriptivas se presentan en la sección tres; en la cuarta sección, se presenta la descripción de la metodología econométrica empleada en el artículo; en la sección cinco, se reportan y discuten los resultados, y, finalmente, la sección seis contiene las conclusiones y recomendaciones de política.

\section{Revisión de la literatura}

Las investigaciones que se han ocupado de analizar la relación entre pobreza y microfinanzas han sido abundantes en los últimos años, y la mayoría se centra en países de Asia y África (Banerjee et al., 2015; Berhane y Gardebroek, 2010; Chowdhury, Ghosh y Wright, 2002; Crepón et al., 2015; Imai et al., 2010; Imai y Azam, 2012; Khandker, 2005; MkNelly y Dunford, 1998). Si bien los trabajos académicos en el tema apuntan a la existencia de efectos potenciales y significativos en dicha relación, no es posible generalizar que la pobreza se reduzca con el acceso y uso de las microfinanzas (Morduch, 1998; Tarozzi et al., 2015).

En un extremo, se encuentran los estudios que identifican efectos positivos de las microfinanzas en la reducción de la pobreza. Por ejemplo, Pitt y Khandker (1998) estimaron el impacto que tiene la participación, por género, en tres de las más grandes instituciones de microfinanzas en Bangladesh y encontraron un efecto positivo en gasto del hogar, activos en posesión de la mujer, oferta de trabajo y asistencia escolar. En un estudio más reciente para este país, Khandker (2005) muestra que la educación de las mujeres determina en gran parte el monto de crédito solicitado por el hogar y que el aumento en el gasto derivado del crédito incrementa la probabilidad de escapar del círculo de la pobreza. En esta misma línea se encuentran las investigaciones realizadas por Imai et al. (2010) en la India y por Ghalib et al. (2015) en Pakistán; en ambos estudios, los autores utilizan técnicas de efectos de tratamiento para estimar el impacto de las microfinanzas en la pobreza. En el primer caso, los resultados muestran que existen efectos positivos en la mayoría de los indicadores de bienestar analizados, mientras que en el segundo estudio se identifican impactos positivos en el gasto en salud, en el ingreso y en la vivienda.

En esta misma línea se ubican trabajos que, a pesar de no concluir de manera contundente que las microfinanzas contribuyen a disminuir la pobreza, sí encuentran efectos positivos en variables relacionadas con el bienestar de los hogares, como son: el consumo de alimentos, la inversión en activos productivos y el gasto en bienes duraderos (Attanasio et al., 2015; Banerjee et al., 2015; Crepón et al., 2015; Karlan y Zinman, 2009). Dentro de este grupo de investigaciones, vale la pena destacar los trabajos de Crepón et al. (2015) y Banerjee et al. (2015), quienes utilizan experimentos aleatorios para controlar el problema de sesgo por selección. 
Por su parte, Crepón et al. (2015) evalúan uno de los programas de microcréditos más grandes de Marruecos e identifican que los créditos incrementan la inversión en activos y los beneficios de sus negocios; sin embargo, dicho incremento se ve contrarrestado por una reducción en los ingresos derivados del trabajo; en conclusión, no existen mejoras en el ingreso neto de los hogares. Por otro lado, Banerjee et al. (2015) realizan un estudio para la ciudad de Hyderabad, en la India, y encuentran que los microcréditos tienen efectos positivos en la inversión y beneficios para los pequeños negocios; así mismo, no encuentran evidencia de cambios importantes en las variables relacionadas con salud, educación y el empoderamiento de las mujeres. Al continuar con el análisis de esta investigación, estos autores encontraron que dos años después, cuando los hogares pertenecientes a las áreas de control tuvieron acceso a los microcréditos y los hogares tratados habían recibido préstamos durante más tiempo y en mayores cantidades, las diferencias entre ambos grupos continuaron siendo poco significativas.

En el otro extremo están aquellos estudios que encuentran un impacto nulo o negativo de las microfinanzas sobre la reducción de la pobreza (George, 2006; Goetz y Gupta, 1996; Morduch, 1998; Tarozzi et al., 2015). En uno de los estudios pioneros en el tema, Morduch (1998) utiliza la misma encuesta que emplearon Pitt y Khandker (1998) e identifica resultados opuestos, pues destaca que las microfinanzas no provocan aumentos significativos en el consumo ni en los niveles de educación de los beneficiarios. En Etiopía, Tarozzi et al. (2015) efectúan un experimento aleatorio para evaluar los impactos de un incremento en el uso de microcréditos, pero, a diferencia del trabajo de Banerjee et al. (2015), en este caso la presencia de otras instituciones microfinancieras antes y durante la realización del experimento fue bastante limitada, razón por la cual los resultados pueden interpretarse como impactos de "primera generación”. Estos autores encuentran que, a pesar del enorme incremento en el número de microcréditos otorgados en las áreas tratadas, no existe un impacto positivo en el ingreso proveniente de actividades agrícolas, crianza de animales, autoempleo no agrícola, oferta de trabajo, escolaridad y empoderamiento de la mujer.

En México, la mayoría de los estudios que han abordado el tema de microfinanzas y pobreza hacen un análisis descriptivo del fenómeno y no emplean metodologías formales que permitan verificar la significancia de los resultados (Cuasquer y Maldonado, 20II; Esquivel, 2010). Sin embargo, Angelucci, Karlan y Zinman (2015) emplean un experimento aleatorio en el norte de México (Sonora) para estimar el impacto, a nivel comunidad, de una expansión en los créditos grupales otorgados por Compartamos Banco, una institución financiera especialista en microfinanzas que opera en México y en América Latina y que cuenta con más de 2,5 millones de clientes, la mayoría de los cuales pertenecen a los segmentos de ingresos bajos de la población (Asociación de Bancos de México, 201I). En este estudio, se estimó el efecto en 37 indicadores distintos, agrupados en seis dimensiones: microemprendimiento, ingreso, oferta de trabajo, consumo, estatus social y bienestar subjetivo. Los resultados muestran evidencia de un incremento en los ingresos de los negocios de los hogares tratados; sin embargo, no identifican efecto en los beneficios. El efecto estimado en los indicadores relacionados con el gasto solo fue estadísticamente significativo en las categorías de gastos en activos y bienes de lujo; vale la pena destacar que la magnitud del efecto, incluso para aquellos indicadores que son significativos, resultó ser bastante pequeña. Así, la conclusión de este trabajo indica que el impacto estimado para la mayoría de las variables analizadas no puede considerarse suficientemente grande como para mejorar las condiciones de vida de los hogares tratados.

\section{Datos y estadísticas descriptivas}

Los datos utilizados para realizar esta investigación provienen de la EnChor 2013 (Coneval, 20I3), cuyo objetivo fundamental consistió en evaluar el desempeño de la Cruzada Nacional Contra el Hambre (Cruzada), recabando información entre noviembre de 2012 y octubre de 20I3; el número total de localidades encuestadas fue de 125 (62 
localidades Cruzada y 63 no Cruzada). Cabe señalar que la Cruzada Nacional Contra el Hambre es una estrategia coordinada por el Gobierno Federal de México cuyo propósito es que las familias superen su condición simultánea de carencia alimentaria y pobreza extrema. Todas las personas que se encuentran en dicha condición constituyen la población objetivo de esta estrategia: en 2010, la cifra ascendía a 7,4 millones de mexicanos distribuidos en todo el país (Coneval, 2017a).

La EnChor 2013 (Coneval, 2013) se recabó mediante dos tipos de cuestionarios: el de hogares y el de localidades. El primero incorporó información acerca de las características socioeconómicas, productivas, financieras y de alimentación de los hogares y, por su parte, el segundo permitió conocer información de las actividades productivas, las obras públicas, los destinos migratorios y los mercados financieros de las localidades. El diseño y la selección de la muestra permitieron obtener resultados representativos para los municipios Cruzada y no Cruzada del país; de esta forma, el tamaño de la muestra fue de 25 II hogares, de los cuales el 9\% reportó haber empleado algún tipo de microcrédito.

La información de la EnChor posibilita la identificación de los usos más frecuentes de los microcréditos en los hogares del sector rural mexicano, dentro de los que se destaca, en primer lugar, la compra, la construcción o el mejoramiento de la vivienda (25\%). El segundo uso más frecuente fue para financiar actividades agropecuarias: el $19 \%$ de los microcréditos otorgados se utilizó con este objetivo. La compra o reparación de maquinaria, autos y locales comerciales representó el I6\%, mientras que el uso en cuestiones de salud y educación fue del iz y el 9\%, respectivamente.

La tabla i resume las estadísticas descriptivas para el total de la muestra, así como para los hogares que utilizaron algún tipo de microcrédito (tratados) y aquellos que no lo hicieron (de control). De igual forma, se presenta la prueba t para la diferencia de medias entre el grupo de hogares tratados (GT) y el grupo de hogares de control (GC), la cual permite verificar si existen disparidades estadísticas en la distribución de cada variable entre el GT y el GC; lo anterior resulta relevante para determinar si la muestra está balanceada y, por lo tanto, si las comparaciones entre ambos grupos están libres de sesgo (Cameron y Trivedi, 20I0). Los resultados de la prueba, reportados en la última columna de la tabla i, muestran que el GT y el GC presentan diferencias estadísticamente significativas en los valores promedio de 9 de las 15 variables consideradas.

$\mathrm{Al}$ respecto de las variables utilizadas en el modelo, autores como MkNelly y Dunford (1999), Chowdhury et al. (2002), Banerjee et al. (2015) y Tarozzi et al. (2015) analizan las características sociodemográficas y productivas del hogar para identificar los factores que determinan el uso y acceso a los créditos; estos autores señalan que entre las características más distintivas de los hogares rurales se encuentran el número de sus integrantes y el número reducido de habitaciones o cuartos en sus viviendas. Al respecto, el tamaño promedio del hogar en la muestra fue de 5,08 integrantes, de los cuales I,4 son niños; por su parte, el número de habitaciones, sin contar la cocina ni el baño, fue de 2,76 (tabla I). Haciendo uso de esta información, se identificaron aquellos hogares que presentan un grado de hacinamiento (número de personas por cuarto) mayor a 2,5; los resultados indican que el $30 \%$ de los hogares analizados poseen esta característica. 
TABLA 1

Estadísticas DEscriptivas

\begin{tabular}{|c|c|c|c|c|c|}
\hline \multirow[t]{2}{*}{ Variable } & \multicolumn{2}{|c|}{ Total de la muestra $^{1}$} & \multicolumn{2}{|c|}{$\begin{array}{l}\text { Medias para los dos } \\
\text { grupos de hogares }\end{array}$} & \multirow[t]{2}{*}{$\begin{array}{l}\text { Prueba } t \\
\text { p-value }\end{array}$} \\
\hline & Media & $\begin{array}{l}\text { Desviación } \\
\text { estándar }\end{array}$ & Tratados $^{1}$ & Controles $^{1}$ & \\
\hline Sexo del jefe del hogar ${ }^{2}$ & 0,81 & 0,39 & 0,85 & 0,81 & $0,091^{*}$ \\
\hline Edad del jefe del hogar & 48,9 & 16,4 & 47,4 & 49,0 & 0,161 \\
\hline $\begin{array}{l}\text { El jefe del hogar habla } \\
\text { alguna lengua indigena }\end{array}$ & 0,22 & 0,41 & 0,11 & 0,23 & $0,000 * * *$ \\
\hline $\begin{array}{l}\text { Educación del jefe del } \\
\text { hogar-primaria completa }\end{array}$ & 0,23 & 0,42 & 0,24 & 0,22 & 0,499 \\
\hline $\begin{array}{l}\text { Educación del jefe del } \\
\text { hogar-secundaria } \\
\text { completa }\end{array}$ & 0,19 & 0,39 & 0,23 & 0,20 & 0,295 \\
\hline $\begin{array}{l}\text { Educación del jefe del } \\
\text { hogar-preparatoria o } \\
\text { superior }\end{array}$ & 0,11 & 0,31 & 0,15 & 0,11 & $0,028^{* *}$ \\
\hline $\begin{array}{l}\text { Número de niños en el } \\
\text { hogar ( }<16 \text { años) }\end{array}$ & 1,40 & 1,42 & 1,61 & 1,38 & $0,022^{* *}$ \\
\hline $\begin{array}{l}\text { Escolaridad más alta de } \\
\text { una mujer adulta en el } \\
\text { hogar }\end{array}$ & 7,86 & 4,24 & 9,39 & 7,72 & $0,000^{* * *}$ \\
\hline $\begin{array}{l}\text { El hogar recibe } \\
\text { transferencias del } \\
\text { Gobiemo }{ }^{2}\end{array}$ & 0,53 & 0,49 & 0,53 & 0,53 & 0,913 \\
\hline $\begin{array}{l}\text { Número de cuartos en el } \\
\text { hogar }\end{array}$ & 2,76 & 1,42 & 3,13 & 2,72 & $0,000^{* * *}$ \\
\hline $\begin{array}{l}\text { Proporción de miembros } \\
\text { del hogar con buena } \\
\text { salud }\end{array}$ & 0,55 & 0,34 & 0,52 & 0,55 & 0,186 \\
\hline $\begin{array}{l}\text { El hogar tiene tierra } \\
\text { agrícola }^{3}\end{array}$ & 0,30 & 0,46 & 0,29 & 0,30 & 0,654 \\
\hline $\begin{array}{l}\text { Superficie de la tierra } \\
\text { agricola }{ }^{4}\end{array}$ & 3,54 & 5,07 & 1,50 & 1,02 & $0,005^{* * *}$ \\
\hline $\begin{array}{l}\text { Instituciones financieras } \\
\text { formales en la localidad }^{3}\end{array}$ & 0,16 & 0,37 & 0,24 & 0,15 & $0,000^{* * *}$ \\
\hline $\begin{array}{l}\text { Prestamistas informales } \\
\text { en la localidad }\end{array}$ & 0,25 & 0,44 & 0,31 & 0,25 & $0,032^{* *}$ \\
\hline
\end{tabular}

Fuente: elaboración propia con base en Coneval (2013)

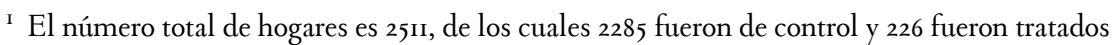

$$
\begin{gathered}
{ }^{2} \mathrm{I}=\text { hombre, } \mathrm{O}=\text { mujer } \\
3 \mathrm{I}=\text { sí, } \mathrm{O}=\text { no }
\end{gathered}
$$

4 Se consideraron únicamente los 755 hogares que reportaron tener tierra agrícola

5 Prueba t para diferencia de medias: ${ }^{* * *},{ }^{* *},{ }^{*}$ : significativas al $\mathrm{I} \%, 5 \%$ y $10 \%$, respectivamente.

En lo que se refiere a las características del jefe del hogar, las cifras señalan que el 23\% de los jefes en el GC reportó hablar alguna lengua indígena, comparado con un iı en el caso del GT; este resultado revela que, en el sector rural mexicano, hablar alguna lengua indígena reduce la probabilidad de tener acceso a las microfinanzas. Por otro lado, el análisis del nivel de educación señala que el 15\% de los jefes en el GT terminó al menos la preparatoria, comparado con el iI \% en el GC.

En relación con las características productivas, el 30\% de los hogares reportaron tener una parcela agrícola, independientemente del tipo de tenencia; además, no se identificó una diferencia estadística en el valor promedio 
de esta variable entre el GT y el GC. Sin embargo, respecto a la superficie promedio, sí existe una diferencia significativa entre ambos grupos, pues es menor la del segundo.

De manera adicional, y siguiendo las recomendaciones de Banerjee et al. (2015) y Tarozzi et al. (2015), para identificar factores adicionales que determinaran el acceso a las microfinanzas, se construyeron las siguientes variables: la escolaridad más alta de una mujer adulta en el hogar, la proporción de miembros del hogar con buena salud y dos variables que indican la existencia de instituciones financieras formales y de prestamistas informales dentro de la localidad. En lo que se refiere a estas variables, es posible señalar que la escolaridad más alta de una mujer adulta en el GT fue de 9,39 años, comparado con 7,72 en el GC, lo cual sugiere que la educación de las mujeres dentro de los hogares rurales juega un papel importante para tener acceso a las microfinanzas, más aún si se considera que la mayoría de los programas que se encargan de otorgar microcréditos están dirigidos a integrantes del hogar del sexo femenino. Por otro lado, el $24 \%$ del GT vive en localidades en donde existe alguna institución financiera formal, mientras que en el GC solo el $15 \%$ tiene acceso a este tipo de instituciones. Así mismo, el $31 \%$ y el $25 \%$ de los hogares tratados y de control tienen, respectivamente, acceso a prestamistas informales dentro de su localidad.

Las diferencias detectadas mediante la prueba t en los valores promedio de algunas variables de interés entre el GT y el GC podrían atribuirse al hecho mismo de utilizar microcréditos; sin embargo, pensar que esto es así no es estadísticamente correcto, y, por lo tanto, es necesario implementar alguna metodología confiable y robusta que permita obtener estimaciones insesgadas entre ambos grupos de hogares, de tal manera que los efectos encontrados puedan efectivamente atribuirse al uso de los microcréditos; es por ello que se emplea la técnica de propensity score matching, expuesta en el siguiente apartado.

\section{Metodología: el propensity score matching (PSM)}

La idea básica para estimar el efecto de las microfinanzas en la pobreza por ingresos de los hogares rurales mexicanos consiste en asumir que utilizar algún microcrédito es como recibir un "tratamiento", de tal forma que el propósito es medir el impacto de dicho tratamiento en cierta variable de interés, en este caso, los niveles de pobreza. Para lograr lo anterior, es necesario calcular la diferencia en la pobreza por ingresos atribuida al hecho de haber recibido el tratamiento, es decir, la diferencia en los niveles de pobreza entre los hogares tratados y los de control.

Una forma de estimar esta diferencia es mediante el enfoque estándar de matching (Rubin, 1973). De acuerdo con este enfoque, la diferencia entre GT y GC puede definirse como:

$$
\Delta_{i}=Y_{i}^{1}-Y_{i}^{0}
$$

donde $_{\Delta_{i}}$ denota el efecto del tratamiento para el hogar i, con $i=1,2, \ldots, N$ mientras que $Y_{i}^{1}$ y $Y_{i}^{0}$ representan los resultados potenciales para tratados y controles, respectivamente. A pesar de no contar con datos panel, y asumiendo que se cumplen los supuestos de exogeneidad y superposición (los cuales se explican más adelante), la ecuación ( $\mathrm{I}$ ) aproxima la diferencia entre los resultados potenciales antes y después de recibir el tratamiento para cada hogar. Cabe destacar que para cada hogar i en la ecuación (I) solo es posible observar un resultado, mientras que el otro es un escenario contrafactual que no se puede obtener con la información disponible.

Por lo tanto, la ecuación (I) debe modificarse de tal forma que permita estimar el efecto promedio del tratamiento en los tratados $\Delta_{A T T}$, el cual puede expresarse de la siguiente manera:

$$
\Delta_{A T T}=E(\Delta \mid D=1)=E\left(Y^{1} \mid D=1\right)-E\left(Y^{0} \mid D=1\right)
$$


donde $\Delta_{A T T}$ mide la diferencia entre el resultado esperado con y sin tratamiento para aquellos hogares que recibieron el tratamiento y donde $D$ es una variable que indica el estado de tratamiento $(D=$ I cuando se recibió y $D=0$ cuando no fue así). Por su parte, el término $E\left(Y^{1} \mid D=1\right)$ representa el resultado esperado para los hogares tratados, mientras que el término $E\left(Y^{0} \mid D=1\right)$ refiere el resultado hipotético que se habría obtenido si los hogares tratados no hubieran recibido el tratamiento.

A partir del análisis de la ecuación (2), es posible destacar la existencia del problema de inobservabilidad, esto es, se puede estimar el término $E\left(Y^{1} \mid D=1\right)$ pero no el término $E\left(Y^{0} \mid D=1\right)$ dado que no es observable. Si la igualdad $E\left(Y^{0} \mid D=0\right)=E\left(Y^{0} \mid D=1\right)$ se cumple, entonces el GC puede usarse convenientemente como grupo de comparación con el GT. Sin embargo, cuando se trabaja con datos no experimentales, como es el caso, esta condición generalmente no se cumple, ya que las variables que determinan la decisión de participación también contribuyen a explicar la variable dependiente. Por consiguiente, el resultado para el GT será distinto incluso en la ausencia del tratamiento, lo que ocasiona un problema de sesgo por autoselección. Por lo tanto, la ecuación (2) puede reexpresarse de la siguiente forma:

$$
E\left(Y^{1} \mid D=1\right)-E\left(Y^{0} \mid D=0\right)=\Delta_{A T T}-\left[E\left(Y^{0} \mid D=0\right)-E\left(Y^{0} \mid D=1\right)\right] \quad(3)
$$

donde $E\left(Y^{0} \mid D=1\right)-E\left(Y^{0} \mid D=0\right)$ mide el tamaño del sesgo ocasionado por todas aquellas características observables. Por lo tanto, el $\Delta_{A T T}$ puede identificarse únicamente cuando el sesgo es cero, es decir, cuando se cumple que:

$$
E\left(Y^{0} \mid D=1\right)-E\left(Y^{0} \mid D=0\right)
$$

Para solucionar este problema, la literatura sugiere implementar procedimientos tales como matching por covariables, o propensity score (Abadie e Imbens, 20II; Rosenbaum y Rubin, 1985; Rubin, 1973).

Ahora bien, como se señaló anteriormente, para obtener estimaciones insesgadas del $\Delta_{A T T}$ es necesario imponer dos supuestos fundamentales: el de exogeneidad del tratamiento y el de superposición.

El supuesto de exogeneidad implica que la diferencia en los resultados potenciales - con y sin tratamiento- se debe únicamente a la implementación del tratamiento condicionada en un vector de características X. Más aún, el conjunto de variables que conforman este vector no se ve afectado por el tratamiento y se asume que ha sido totalmente capturado en el modelo, es decir, que no existen variables omitidas. Formalmente, este supuesto se define de la siguiente manera:

\section{Supuesto I $\left\{Y^{0}, Y^{1}\right\} \perp D \mid \mathbf{X}(5)$}

El segundo supuesto garantiza que todos los hogares con las mismas características dentro de la muestra tengan una probabilidad positiva de ser tratados o controles y, además, descarta la perfecta previsibilidad de participación condicional en el vector de características X. Esta condición se define como:

$$
\text { Supuesto 2: } 0<P(D=1 \mid \mathbf{X})<1
$$

Si estos dos supuestos se cumplen, es posible estimar la ecuación (2) utilizando propensity score matching (PSM), una técnica que permite resolver el problema de dimensionalidad que surge cuando el vector de características observables X tiene demasiados componentes. Para ello, se utiliza lo que se conoce como puntaje de equilibrio o propensity score, sugerido por primera vez por Rosenbaum y Rubin (1983) y que se define como una función que estima la probabilidad de recibir el tratamiento dado un vector de características previamente observadas X; es decir, permite resumir el vector de características $\mathrm{X}$ en un solo número $\tau(\mathbf{X})$. Formalmente, el propensity score se define como:

$$
P(D=1 \mid \mathbf{X})=\tau(\mathbf{X}) \quad(7)
$$

El propensity score puede estimarse utilizando los modelos probit o logit, ya que ambos modelos permiten predecir la probabilidad de que un hogar haya utilizado algún tipo de microcrédito basándose únicamente en sus propias características. Tomando esto en consideración, la ecuación (7) puede expresarse de la siguiente forma:

$$
P(D=1 \mid \mathbf{X})=P\left(y^{*}>0 \mid \mathbf{X}\right)=P(u>-\mathbf{X} \beta \mid \mathbf{X})=1-\Phi(-\mathbf{X} \beta)=\Phi(\mathbf{X} \beta)
$$

donde $0<\Phi(\mathbf{X} \beta)<1$ para todos los valores de $\mathrm{X} ; \quad y^{*}=\mathbf{X} \beta+u ; y^{*}=\mathbf{X} \beta+u$ y $\Phi$ es la función de distribución acumulada de una normal estándar. 
Si la ecuación (8) satisface el supuesto de exogeneidad y el de superposición, el estimador de PSM para $\Delta_{A T T}$ no presenta problemas de sesgo por autoselección y puede definirse formalmente como:

$$
\Delta_{A T T}^{P S M}=E_{\tau(\mathbf{X}) \mid D=1}\left[E\left(Y^{1} \mid D=1, \tau(\mathbf{X})\right)-E\left(Y^{0} \mid D=1, \tau(\mathbf{X})\right)\right] \quad(9)
$$

Existen distintos algoritmos que la literatura académica utiliza para hacer el matching y estimar el $\Delta_{A T T}$ en este trabajo se emplean cinco algoritmos: nearest neighbor, radius, kernel, local linear regression y stratification (Becker e Ichino, 2002; Imbens, 2000; Todd, 2010). El uso de distintos algoritmos permite comparar resultados y al mismo tiempo probar qué tan robustas son las estimaciones; adicionalmente, en el Apéndice A se presentan las pruebas de balance por bloque y por variable y la prueba de balance general, lo que proporciona evidencia suficiente de la correcta especificación del modelo empleado (Cameron y Trivedi, 2010).

\section{Resultados}

\section{Estimaciones de pobreza por ingresos}

En la tabla 2 se presentan las estimaciones de pobreza para el total de hogares de la muestra, el GT y el GC; estos cálculos incluyen tres indicadores de la familia de medidas de pobreza $P_{\alpha}(\alpha=0,1,2) \quad$ (Foster, Greer y Thorbecke, 1984) y consideran las dos líneas de bienestar oficiales en México (bienestar y bienestar mínimo). La primera de ellas posibilita la identificación de aquellos hogares que no cuentan con los recursos suficientes para satisfacer sus necesidades alimentarias y no alimentarias; por su parte, la línea de bienestar mínimo permite identificar los hogares que, aun al hacer uso de todos sus recursos en la compra de alimentos, no pueden adquirir lo indispensable para tener una nutrición adecuada (Coneval, 2009).

Los resultados muestran que el número de pobres $\left(P_{0}\right)$ es del $47 \%$ utilizando la línea de bienestar mínimo y se incrementa al $76 \%$ si se incluye el valor de la canasta no alimentaria; en ambos casos, la proporción de pobres es mayor en los hogares que no utilizaron algún microcrédito. Por su parte, el índice de pobreza Foster-GreerThorbecke (FGT) $\left(P_{2}\right)$ es de o,12 cuando se toma como referencia la línea de bienestar mínimo y aumenta a 0,26 al considerar la línea de bienestar; una vez más, el indicador de pobreza es mayor en aquellos hogares que no usaron microcréditos. Las cifras de la tabla 2 señalan que tanto el número de pobres $P_{0}$ como la profundidad y severidad de la pobreza $\left(P_{1}\right.$ y $\left.P_{2}\right)$ son mayores para los hogares que están aislados o no participan en los mercados financieros disponibles en el sector rural de México.

TABLA 2

EstimACIONES DE POBREZA

\begin{tabular}{ccccccc}
\hline \multirow{2}{*}{$\begin{array}{c}\text { Índice } \\
F G T_{\alpha}\end{array}$} & \multicolumn{2}{c}{ Línea de bienestar mínimo ${ }^{\mathbf{1}}$} & \multicolumn{3}{c}{ Línea de bienestar $^{\mathbf{2}}$} \\
\cline { 2 - 7 } & $\begin{array}{c}\text { Total de } \\
\text { hogares }\end{array}$ & Tratados & Controles & $\begin{array}{c}\text { Total de } \\
\text { hogares }\end{array}$ & Tratados & Controles \\
\hline$(\alpha=0)$ & 0,47 & 0,27 & 0,49 & 0,76 & 0,61 & 0,78 \\
\hline$(\alpha=1)$ & 0,20 & 0,10 & 0,21 & 0,4 & 0,27 & 0,42 \\
\hline$(\alpha=2)$ & 0,12 & 0,05 & 0,13 & 0,26 & 0,15 & 0,27 \\
\hline
\end{tabular}

Fuente: elaboración propia con base en Coneval (2013)

${ }^{\text {I }}$ Cálculos elaborados con una línea de bienestar mínimo de \$9976,5 para el periodo noviembre de 20I2-octubre de 2013

${ }^{2}$ Cálculos elaborados con una línea de bienestar de \$18.517,5 para el periodo noviembre de 20I2-octubre de 2013 


\section{Resultados con propensity score matching (PSM)}

Una vez asegurado que el GC está construido de manera adecuada, es posible obtener estimaciones confiables e insesgadas del $\Delta_{A T T}$; de esta forma, los resultados que se presentan a continuación proporcionan evidencia sólida de que las diferencias entre los dos grupos de hogares no son estocásticas, sino que pueden atribuirse al tratamiento recibido, en nuestro caso, al uso de algún microcrédito.

El primer paso para presentar las estimaciones del $\Delta_{A T T}$ mediante PSM consiste en mostrar los resultados del modelo probit, que identifica las características de los hogares que determinan el uso de microcréditos (tabla 3 ).

Dentro de los resultados más destacados, se encuentran los siguientes. En primer lugar, la probabilidad de que los hogares utilicen un microcrédito es menor para aquellos en los cuales el jefe habla alguna lengua indígena; en contraste, dicha probabilidad aumenta conforme se incrementa el número de niños en el hogar, resultado que probablemente se encuentre relacionado con el hecho de que en aquellos hogares con más niños se utilizan recursos monetarios con mayor frecuencia para solventar gastos en salud y educación. Por su parte, la probabilidad de que un hogar utilice un microcrédito está positivamente relacionada con la escolaridad más alta de una mujer adulta en el hogar; esto refleja el hecho de que el nivel de educación de las mujeres juega un papel importante en el tipo de decisiones financieras que se toman al interior del hogar. Así mismo, se encuentra evidencia de que los hogares con un mayor número de cuartos son más propensos a utilizar algún microcrédito y de que, mientras mayor sea la proporción de miembros del hogar con buena salud, la probabilidad de utilizar algún microcrédito disminuye. Finalmente, los resultados sugieren que aquellos hogares que radican en localidades donde existen instituciones financieras formales tienen mayor probabilidad de utilizar algún tipo de préstamo. 
TABLA 3

RESULTADOS DEL MODELO PROBIT: DETER MINANTES DEL USO DE MICROCRÉDITO RURAL

Variables explicativas

\section{Efectos marginales}

\begin{tabular}{|c|c|}
\hline \multirow[t]{2}{*}{ Sexo del jefe del hogar } & 0,01889 \\
\hline & $(1,3)$ \\
\hline \multirow[t]{2}{*}{ Edad del jefe del hogar } & 0,00035 \\
\hline & $(0,18)$ \\
\hline \multirow{2}{*}{$\begin{array}{l}\text { Edad del jefe del hogar al } \\
\text { cuadrado }\end{array}$} & $-0,000006$ \\
\hline & $(-0,32)$ \\
\hline \multirow{2}{*}{$\begin{array}{l}\text { El jefe del hogar habla } \\
\text { alguna lengua indigena }\end{array}$} & $-0,0467$ \\
\hline & $(-3,00)^{* * * *}$ \\
\hline \multirow{2}{*}{$\begin{array}{l}\text { Educación del jefe del hogar } \\
\text { (primaria completa) }\end{array}$} & 0,0091 \\
\hline & $(0,63)$ \\
\hline \multirow{2}{*}{$\begin{array}{l}\text { Educación del jefe del hogar } \\
\text { (secundaria completa) }\end{array}$} & $-0,0003$ \\
\hline & $(-0,02)$ \\
\hline \multirow{2}{*}{$\begin{array}{l}\text { Educación del jefe del hogar } \\
\text { (preparatoria o superior) }\end{array}$} & 0,0067 \\
\hline & $(0,35)$ \\
\hline \multirow{2}{*}{$\begin{array}{l}\text { Número de niños en el hogar } \\
\text { ( }<16 \text { años })\end{array}$} & 0,0092 \\
\hline & $(2,14)^{* * *}$ \\
\hline \multirow{2}{*}{$\begin{array}{l}\text { Escolaridad más alta de una } \\
\text { mujer adulta en el hogar }\end{array}$} & 0,0064 \\
\hline & $(4,50) * * *$ \\
\hline \multirow{2}{*}{$\begin{array}{l}\text { E1 hogar recibe } \\
\text { transferencias del Gobierno }\end{array}$} & 0,0142 \\
\hline & $(1,19)$ \\
\hline \multirow{2}{*}{$\begin{array}{l}\text { Número de cuartos en el } \\
\text { hogar ( sin contar cocina y } \\
\text { baño) }\end{array}$} & 0,0109 \\
\hline & $(2,91)^{* * * *}$ \\
\hline \multirow{2}{*}{$\begin{array}{l}\text { Proporción de miembros del } \\
\text { hogar con buena salud }\end{array}$} & $-0,0276$ \\
\hline & $(-1,67)^{*}$ \\
\hline \multirow[t]{2}{*}{ El hogar tiene tierra agrícola } & $-0,0096$ \\
\hline & $(-0,65)$ \\
\hline \multirow{2}{*}{$\begin{array}{l}\text { Superficie de la tierra } \\
\text { agrícola }\end{array}$} & 0,0034 \\
\hline & $(2,11)^{* * *}$ \\
\hline \multirow{2}{*}{$\begin{array}{l}\text { Instituciones financieras } \\
\text { formales en la localidad }\end{array}$} & 0,0444 \\
\hline & $(3,24) * * *$ \\
\hline \multirow{2}{*}{$\begin{array}{l}\text { Prestamistas informales en la } \\
\text { localidad }\end{array}$} & 0,0097 \\
\hline & $(0,79)$ \\
\hline
\end{tabular}

Fuente: elaboración propia con base en Coneval (2013)

Nota: los números entre paréntesis son los valores z: ${ }^{* * *},{ }^{* *},{ }^{*}$ : significativos al $1 \%, 5 \%$ y $10 \%$, respectivamente. 
Después de analizar los resultados del modelo probit, que identifican los determinantes del uso de microcréditos en el sector rural mexicano, el siguiente paso consistió en verificar la existencia de un área de soporte común que garantice estimaciones confiables. La figura i muestra el histograma del GT y el GC que surge a partir de la estimación del modelo probit antes descrito; dicha figura muestra claramente que, para valores del propensity score entre $\diamond, \circ$ y $\circ, 3$, existe un traslape importante en la distribución de ambos grupos, lo que indica que el supuesto de superposición se cumple. Para reforzar el argumento anterior, es posible señalar que las pruebas de balance por bloque y por variable y la prueba de balance general (Apéndice A, tablas $A_{1}, A_{2}, A_{3}$ ) demuestran que, al condicionar en los propensity scores, no hay diferencias estadísticamente significativas entre el GT y el GC y que, por lo tanto, es posible obtener estimaciones confiables atribuidas al efecto del tratamiento.

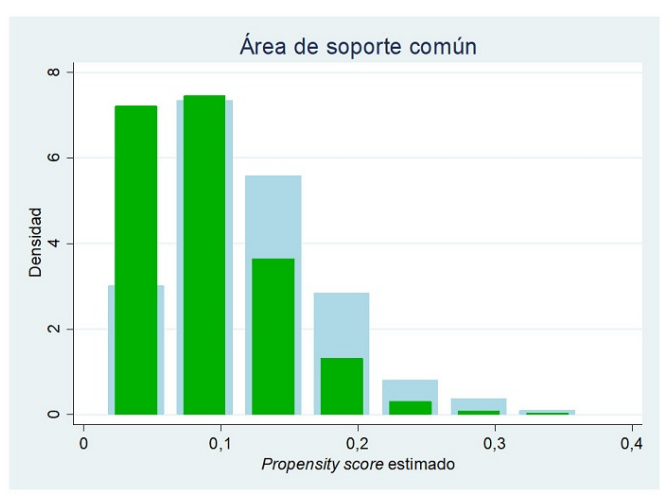

FIGURA 1

Histograma del propensity score para el GT y el GC

Nota: el histograma de color verde corresponde al GC, y el de color azul se refiere al GT.

Fuente: elaboración PRopia con Base en ConeVal (2O13)

En la tabla 4 se presentan las estimaciones del $\Delta_{\text {ATr }}$ para cinco algoritmos de matching: nearest neighbor, stratification, kernel, local linear regression y radius; para aquellos algoritmos en los que no se calcula el error estándar de forma directa, se utilizó el método bootstrap (Becker e Ichino, 2002).

TABLA 4

EFEcto PROMEdio DEL TRATAMIENTO EN LOS TRATAdos (ATT): PROPENSITY SCORE MATCHING

\begin{tabular}{|c|c|c|c|c|c|}
\hline \multirow{2}{*}{$\begin{array}{c}\text { Variable } \\
\text { dependiente }\end{array}$} & \multicolumn{5}{|c|}{ Algoritmo utilizado para hacer el matching } \\
\hline & $\begin{array}{c}\text { Nearest } \\
\text { neighbor }\end{array}$ & Stratification & Kernel & Radius & $\begin{array}{c}\text { Local linear } \\
\text { regression }\end{array}$ \\
\hline \multirow{2}{*}{$\begin{array}{c}\text { Ingreso } \\
\text { (línea de } \\
\text { bienestar) }\end{array}$} & $-13,7$ & $-14,9$ & -16 & $-15,9$ & $-14,9$ \\
\hline & $(-2,99)^{* * * *}$ & $(-4,30)^{* * * *}$ & $\begin{array}{c}(- \\
4,93)^{* * * *}\end{array}$ & $\begin{array}{c}(- \\
4,56)^{* * * *}\end{array}$ & $(-3,52)^{* * * *}$ \\
\hline \multirow{2}{*}{$\begin{array}{l}\text { Ingreso } \\
\text { (línea de } \\
\text { bienestar } \\
\text { mínimo) }\end{array}$} & $-13,7$ & $-17,1$ & $-19,3$ & $-20,4$ & $-17,7$ \\
\hline & $(-2,91)^{* * * *}$ & $(-5,37) * * * *$ & $\frac{(-}{6,32)^{* * * *}}$ & $\begin{array}{c}(- \\
6,17)^{* * * *}\end{array}$ & $(-3,75)^{* * * *}$ \\
\hline
\end{tabular}

El valor del estadístico t de Student se muestra entre paréntesis: ***, ** , *: significativos al I\%, $5 \%$ y 10\%, respectivamente.

Fuente: elaboración propia con base en Coneval (2013)

Nota: Las cifras de los efectos representan puntos porcentuales.

Los efectos mostrados en la tabla 4 corresponden a cambios porcentuales en la probabilidad de que un hogar sea pobre por ingresos (utilizando como umbral la línea de bienestar o la línea de bienestar mínimo) por efecto del uso de un microcrédito. Al emplear la línea de bienestar, se encontró que utilizar algún microcrédito disminuye en promedio un $15 \%$ la probabilidad de que un hogar se encuentre en situación de pobreza por ingresos; dependiendo 
del algoritmo de matching empleado, este resultado varía desde un mínimo de $13,7 \%$, cuando se usa nearest neighbor, hasta un máximo de $16 \%$, cuando el algoritmo empleado es kernel.

Ahora bien, cuando se fija como umbral de pobreza la línea de bienestar mínimo, los resultados refuerzan lo antes señalado; en este caso, la probabilidad de que un hogar se considere pobre disminuye en promedio un 17,6\%. Con este umbral de pobreza, el rango de variación del $\Delta_{\text {art }}$ es más amplio que en el caso anterior, pues va desde 13,7\%, cuando se emplea nearest neighbor, hasta un $20,4 \%$, cuando el algoritmo utilizado es radius.

Estos hallazgos concuerdan con los de MkNelly y Dunford (1999), quienes encuentran un resultado similar cuando estiman el impacto que tienen los servicios de crédito en los ahorros y el ingreso de las mujeres beneficiarias del programa Crecer en Bolivia. Así mismo, respaldan lo encontrado por Imai y Azam (20I2) en Bangladesh y Ghalib et al. (2015) en Pakistán, investigaciones que también identifican impactos positivos en el ingreso, gasto y consumo de los hogares beneficiarios de microcréditos. No obstante, contrastan con los trabajos de Crepón et al. (2015) y Angelucci et al. (2015), quienes atribuyen efectos nulos o negativos para variables relacionadas con el ingreso de los hogares.

Una posible explicación de los resultados presentados en la tabla 4 es que los microcréditos otorgados a los hogares aumentan su ingreso disponible, lo que a su vez provoca que aumente el gasto per cápita y, por lo tanto, que la probabilidad de que este sea menor a la línea de bienestar o bienestar mínimo disminuya. Sin embargo, si adoptamos como válida esta explicación, es importante cuestionar si este efecto en la pobreza es de largo plazo o si se trata solamente de un efecto temporal derivado del uso de microcréditos; desafortunadamente, los datos de la EnChor 2013 (Coneval, 2013) no permiten responder esta pregunta, y para ello sería deseable contar con datos tipo panel que posibiliten analizar la dinámica de estos efectos. A pesar de este señalamiento, es posible apuntar que en todos los casos del matching empleado, e independientemente de la línea de bienestar utilizada, los efectos en la reducción de la pobreza atribuidos al uso de los microcréditos van en la misma dirección, lo cual es evidencia de la robustez y consistencia de los hallazgos encontrados en la presente investigación.

\section{Conclusiones}

Utilizando datos de la Encuesta Coneval a Hogares Rurales de México 2013, en este estudio se estimó el impacto que tiene el uso de las microfinanzas en la pobreza por ingresos de los hogares rurales de México. Para controlar el problema de sesgo por autoselección que surge cuando se trabaja con datos no experimentales, se utilizó propensity score matching (PSM), metodología que permite comparar el resultado alcanzado en la variable dependiente entre el GT y el GC. Con la finalidad de generar robustez en los resultados, el efecto promedio del tratamiento en los tratados ( $\left.\Delta_{\text {Art }}\right)$ se estimó utilizando cinco diferentes algoritmos de matching.

Los resultados encontrados permiten identificar que el uso de microcréditos disminuye la probabilidad de que

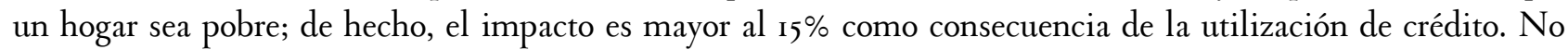
obstante, esta reducción puede no (o sí) estar ligada con una mejora en las condiciones de vida permanentes de los hogares rurales, sino únicamente estar reflejando el aumento en el ingreso como consecuencia del monto del crédito otorgado.

A pesar de las limitaciones en la metodología de PSM, como puede ser la imposibilidad de observar todas las variables que determinan el uso de los microcréditos, los resultados encontrados en esta investigación permiten sugerir medidas de política enfocadas en facilitar el uso de crédito en los hogares rurales pobres. Promover de manera sostenida este tipo de políticas permitiría reducir los niveles de pobreza que enfrenta una proporción considerable de los hogares rurales de México y, como consecuencia, impulsaría el acceso a mayores oportunidades laborales para los integrantes de dichos hogares. 
De forma complementaria a esta investigación, se recomienda el uso de fuentes alternativas de información que permitan verificar si los efectos estimados en este trabajo son de carácter permanente o temporal. Por lo anterior, las conclusiones de este artículo no pueden ni deben tomarse como definitivas y están susceptibles de mejoras mediante la aplicación de otro tipo de metodologías.

Finalmente, resulta fundamental señalar que, para poder diseñar políticas o programas que realmente atiendan las necesidades de los hogares rurales que se encuentran en situación de pobreza, es necesario hacer caso a las recomendaciones que investigadores como Abhijit Banerjee y Esther Duflo hacen al respecto, en el sentido de que primero debemos interesarnos en conocer a fondo la forma en la que estos hogares estructuran sus decisiones (financieras, laborales, de salud, entre otras) para posteriormente proponer medidas adecuadas con base en los conocimientos previamente adquiridos.

\section{Referencias}

Abadie, A., e Imbens, G. W. (20II). Bias-corrected matching estimators for average treatment effects. Journal of Business and Economic Statistics, 29(I), I-II. https://www.doi.org/I0.II98/jbes.2009.07333

Angelucci, M., Karlan, D., y Zinman, J. (2015). Microcredit impacts: Evidence from a randomized microcredit program placement experiment by Compartamos Banco. American Economic Journal: Applied Economics, 7(I), I5I-I82. Recuperado de https://www.nber.org/papers/wi9827

Asociación de Bancos de México. (20II). Anuario financiero de la banca en México 20II. Recuperado de https:// www.abm.org.mx/anuario/anuario2oı/pdf/compartamos-banco.pdf

Attanasio, O., Augsburg, B., de Haas, R., Fitzsimons, E., y Harmgart, H. (2015). The impacts of microfinance: Evidence from joint-liability lending in Mongolia. American Economic Journal: Applied Economics, 7(I), 90-122. https://www.dx.doi.org/10.2139/ssrn.1974414

Banco Mundial. (2017). Monitoring global poverty: Report of the Commission on Global Poverty. Washington: autor. Recuperado de https://www.openknowledge.worldbank.org/handle/ro986/25I4I

Banerjee, A., Duflo, E., Glennerster, R., y Kinnan, C. (2015). The miracle of microfinance? Evidence from a randomized evaluation. American Economic Journal: Applied Economics, 7(I), 22-53. https://www.doi.org $/$ I0.3386/wi8950

Banerjee, A. V., y Duflo, E. (20II). Poor economics: A radical rethinking of the way to fight global poverty. Public Affairs.

Banerjee, S. B., y Jackson, L. (2017). Microfinance and the business of poverty reduction: Critical perspectives from rural Bangladesh. Human Relations, 70(I), 63-91. https://www.doi.org/10.1177/0018726716640865

Bateman, M. (20II). Microfinance as a development and poverty reduction policy: Is it everything it's cracked up to be? Londres: Overseas Development Institute.

Becker, S. O., e Ichino, A. (2002). Estimation of average treatment effects based on propensity scores. The Stata Journal, 2(4), 358-377. Recuperado de https://www.econpapers.repec.org/article/tsjstataj/v_3a2_3ay_3a2002_ 3ai_3a4_3ap_3a358-377.htm

Berhane, G., y Gardebroek, C. (2010). Does microfinance reduce rural poverty? Evidence based on household panel data from Northern Ethiopia. American Journal of Agricultural Economics, 93(I), 43-55. https://www.doi .org/10.1093/ajae/aaq126

Caliendo, M., y Kopeinig, S. (2008). Some practical guidance for the implementation of propensity score matching. Journal of Economic Surveys, 22(I), 3I-72. Recuperado de https://www.iza.org/publications/dp/1588/somepractical-guidance-for-the-implementation-of-propensity-score-matching 
Cameron, A. C., y Trivedi, P. K. (20ro). Microeconometrics using Stata: Revised edition (2.a). Stata Press.

Chowdhury, M., Ghosh, D., y Wright, R. (2002). The impact of micro-credit on poverty: Evidence from Bangladesh. Progress in Development Studies, 5(4), 298-309. https://www.doi.org/I0.I191/I464993405psir6oa

Consejo Nacional de Evaluación de la Política de Desarrollo Social [Coneval]. (2009). Metodología para la medición multidimensional de la pobreza en México. Ciudad de México: autor. Recuperado de https://ww w.coneval.org.mx/rw/resource/Metodologia_Medicion_Multidimensional.pdf

Consejo Nacional de Evaluación de la Política de Desarrollo Social [Coneval]. (2013). Encuesta Coneval a Hogares Rurales de México 2013. Recuperado de https://www.coneval.org.mx/Informes/Evaluacion/ENCHOR_2 013/ENCHOR_2013_DTA/ENCHOR_2013_DTA.zip

Consejo Nacional de Evaluación de la Política de Desarrollo Social [Coneval]. (2017a). Esquema General de Evaluación de la Cruzada Nacional Contra el Hambre, 2013-2018. Ciudad de México: autor. Recuperado de https://www.coneval.org.mx/Informes/Evaluacion/Cruzada\%20contra\%20el\%20Hambre/ ESQUEMA_GENERAL_DE_EVALUACION_DE_LA_CNCH_\% $20 \mathrm{~F}$.pdf

Consejo Nacional de Evaluación de la Política de Desarrollo Social [Coneval]. (2017b). Medición de la pobreza en México y en las Entidades Federativas 20r6. Ciudad de México: autor.

Crepón, B., Devoto, F., Duflo, E., y Pariente, W. (2015). Estimating the impact of microcredit on those who take it up: Evidence from a randomized experiment in Morocco. American Economic Journal: Applied Economics, 7(I), I23-150. https://www.doi.org/I0.1257/app.20130535

Cuasquer, H., y Maldonado, R. (20II). Microfinanzas y microcrédito en Latinoamérica. Estudios de caso: Colombia, Ecuador, El Salvador, México y Paraguay. Centro de Estudios Monetarios Latinoamericanos: Asociación Regional de Bancos Centrales, Documento de Discusión n.o 2. Recuperado de https://www.id eas.repec.org/p/cml/docdsc/2.html

Esquivel, H. (2010). Medición del efecto de las microfinanzas en México. Comercio Exterior, 6(I), 9-27. Recuperado de https://www.revistas.bancomext.gob.mx/rce/magazines/134/I/09_ESQUIVEL_microfinanzas.pdf

Foster, J., Greer, J., y Thorbecke, E. (1984). A class of decomposable poverty measures. Econometrica, 52(3), 761-766. https://www.doi.org/10.2307/1913475

Fosu, A. K. (2017). Growth, inequality, and poverty reduction in developing countries: Recent global evidence. Research in Economics, 71(2), 306-336. https://www.doi.org/10.1016/j.rie.2016.05.005

George, A. (2006). Why the fight against poverty is failing: A contrarian view. Knowledge Knowledge@SMU (Singapore Management University). Recuperado de https://www.ink.library.smu.edu.sg/cgi/viewcontent .cgi?article=1022\&context=ksmu

Ghalib, A., Malki, I., e Imai, K. (2015). Microfinance and household poverty reduction: Empirical evidence from rural Pakistan. Oxford Development Studies, 43(I), 84-I04. https://www.doi.org/I0.1080/13600818.2014.9802 28

Goetz, A. M., y Gupta, R. S. (1996). Who takes the credit? Gender, power, and control over loan use in rural credit programs in Bangladesh. World Development, 24(I), 45-63. https://www.doi.org/10.1016/0305-750X(95)0012 $4-\mathrm{U}$

Imai, K., Arun, T., y Annim, S. (2010). Microfinance and household poverty reduction: New evidence from India. World Development, 38(I2), I760-I774. https://www.doi.org/I0.I0I6/j.worlddev.2010.04.006

Imai, K., y Azam, S. (20I2). Does microfinance reduce poverty in Bangladesh? New evidence from household panel data. The Journal of Development Studies, 48(5), 633-653. https://www.doi.org/10.1080/00220388.2012.661853 
Imbens, G. W. (2000). The role of the propensity score in estimating dose-response functions. Biometrika, 87(3), 706-710. https://www.doi.org/10.3386/to237

Karlan, D., y Zinman, J. (2009). Expanding microenterprise credit access: Using randomized supply decisions to estimate the impacts on Manila. Centre for Economic Policy Research (CEPR), Discussion Paper n.o $\mathrm{DP}_{7396}$. Recuperado de https://www.papers.ssrn.com/sol3/papers.cfm?abstract_id=I469875

Kasali, T., Ahmad, S., y Ean, L. (2015). Does microfinance operation have effect on poverty alleviation in Nigeria? European Journal of Contemporary Economics and Management, 2(2), 54-69. https://www.dx.doi.org/I0.I 9044/elp.v2no2a4

Khandker, S. (2005). Microfinance and poverty: Evidence using panel data from Bangladesh. The World Bank Economic Review, 19(2), 263-286. Recuperado de https://www.documents.worldbank.org/curated/en/28480 I468013215718/Microfinance-and-poverty-evidence-using-panel-data-from-Bangladesh

Khandker, S., y Samad, H. A. (2018). Bangladesh's achievement in poverty reduction: The role of microfinance revisited. En Y. Sawada, M. Mahmud y N. Kitano (eds.), Economic and social development of Bangladesh (pp. 177-198). Japón: Palgrave MacMillan.

MkNelly, B., y Dunford, C. (1998). Impact of credit with education on mothers and their young children's nutrition: Lower Pra Rural Bank credit with education program in Ghana. Freedom from Hunger, Research Paper n.o 4. Recuperado de https://www.freedomfromhunger.org/impact-credit-education-mothers-and-their-youngchildren $\% \mathrm{E}_{2} \% 80 \%$ 99s-nutrition-lower-pra-rural-bank-०

MkNelly, B., y Dunford, C. (1999). Impacto de crédito con educación en las madres y en la nutrición de sus niños pequeños: programa Crecer de crédito con educación en Bolivia. Freedom from Hunger, Trabajo de Investigación n.o 5. Recuperado de https://www.freedomfromhunger.org/sites/default/files/R5_Bolivia_I mpact_Study_Exec_Summary_8-99-spa.pdf

Morduch, J. (1998). Does microfinance really help the poor? New evidence from flagship programs in Bangladesh. Princeton University, Woodrow Wilson School of Public and International Affairs, Research Program in Development Studies, Working Paper n.o 198. Recuperado de https://www.wagner.nyu.edu/impact/researc $\mathrm{h}$ /publications/does-microfinance-really-help-poor-new-evidence-flagship-programs

Patel, R., Patel, M., y Patel, N. (2018). Impact of microfinance on poor women: Lessons from North Gujarat. Prabandhan: Indian Journal of Management, II(2). https://www.doi.org/I0.17010/pijom/2018/viri2/I21393

Pitt, M., y Khandker, S. (1998). The impact of group-based credit programs on poor households in Bangladesh: Does the gender of participants matter? Journal of Political Economy, 106(5), 958-996. https://www.doi.org $/ 10.1086 / 250037$

Rosenbaum, P., y Rubin, D. (1983). The central role of the propensity score in observational studies for causal effects. Biometrika, 70(I), 4I-55.

Rosenbaum, P., y Rubin, D. (1985). Constructing a control group using multivariate matched sampling methods that incorporate the propensity score. The American Statistician, (39), 33-38. https://doi.org/10.1093/ biomet/70.I.4I

Rubin, D. B. (1973). Matching to remove bias in observational studies. Biometrics, 29(I), I59-I83. https://www.d oi.org/10.2307/2529684

Sani, A., Khan, M. S., Ahmed, H. R. N., y Aziz, B. (2017). Role of micro finance institutions in poverty reduction. Imperial Journal of Interdisciplinary Research, 3(2), 209-212. Recuperado de https://www.onlinejournal.in/ IJIRV3I2/036.pdf

Sultana, H. Y., Jamal, M. A., y Najaf, D.-E. (2017). Impact of microfinance on women empowerment through poverty alleviation: An assessment of socioeconomic conditions in Chennai City of Tamil Nadu. Asian 
Journal for Poverty Studies, 3(2), 175-I83. Recuperado de https://www.ejournal.unib.ac.id/index.php/ajps/a rticle/view/2785

Tarozzi, A., Desai, J., y Johnson, K. (2015). The impacts of microcredit: Evidence from Ethiopia. American Economic Journal: Applied Economics, 7(I), 54-89. https://www.doi.org/I0.1257/app.20130475

Todd, P. E. (2010). Matching estimators. En S. N. Durlauf y L. E. Blume (eds.), Microeconometrics, (pp. I08-I2I). Reino Unido: Palgrave MacMillan.

Apéndice A. Pruebas de balance

TABLA A.l

Prueba de balance por bloque

\begin{tabular}{|c|c|c|c|}
\hline & Observaciones & Media & $\begin{array}{c}\text { Error } \\
\text { estándar }\end{array}$ \\
\hline \multicolumn{4}{|c|}{$\begin{array}{l}\text { Bloque } 1: 0,0133 \leq \text { Score }_{\text {probit }} \leq \\
0,05\end{array}$} \\
\hline Controles & 557 & 0,0339 & 0,0004 \\
\hline Tratados & 20 & 0,0366 & 0,0023 \\
\hline Combinado & 577 & 0,0340 & 0,0004 \\
\hline Diferencia & & $-0,0027$ & 0,0023 \\
\hline Prueba (p-value) & & 0,2567 & \\
\hline \multicolumn{4}{|c|}{ Bloque $2: 0,05 \leq$ Score $_{\text {probit }} \leq 0,1$} \\
\hline Controles & 904 & 0,0737 & 0,0004 \\
\hline Tratados & 61 & 0,0764 & 0,0019 \\
\hline Combinado & 965 & 0,0739 & 0,0005 \\
\hline Diferencia & & $-0,0027$ & 0,0019 \\
\hline Prueba (p-value) & & 0,146 & \\
\hline \multicolumn{4}{|c|}{ Bloque $3: 0,1 \leq$ Score $_{\text {probit }} \leq 0,2$} \\
\hline Controles & 697 & 0,1355 & 0,0010 \\
\hline Tratados & 123 & 0,1352 & 0,0025 \\
\hline Combinado & 820 & 0,1354 & 0,0009 \\
\hline Diferencia & & 0,0002 & 0,0026 \\
\hline Prueba ( $p$-value $)$ & & 0,9315 & \\
\hline \multicolumn{4}{|c|}{ Bloque $4: 0,2 \leq$ Score $_{\text {probit }} \leq 0,3536$} \\
\hline Controles & 72 & 0,2307 & 0,0037 \\
\hline Tratados & 22 & 0,2419 & 0,0085 \\
\hline Combinado & 94 & 0,2333 & 0,0035 \\
\hline Diferencia & & $-0,0113$ & 0,0082 \\
\hline Prueba (p-value) & & 0,1738 & \\
\hline
\end{tabular}

Fuente: elaboración propia con base en Coneval (2013)

Nota: se está probando la hipótesis nula de que no hay diferencia entre tratados y controles contra la alternativa de que sí existe diferencia. Todos los cálculos se realizaron utilizando la función pscore de Stata, desarrollada por Becker e Ichino (2002). En nuestro caso, el número estimado de bloques para remover el sesgo fue 4 . 
TABLA A.2

Prueba de balance por Variable

\begin{tabular}{|c|c|c|c|c|c|}
\hline \multirow[t]{2}{*}{ Variable } & \multirow[t]{2}{*}{ Muestra $^{1}$} & \multicolumn{2}{|c|}{ Media } & \multirow[b]{2}{*}{ Estadístico $t$} & \multirow[b]{2}{*}{ p-value } \\
\hline & & Tratados & Controles & & \\
\hline \multirow[t]{2}{*}{ Sexo del jefe del hogar } & $\mathrm{A}$ & 0,85398 & 0,80799 & 1,69 & $0,091^{*}$ \\
\hline & $\mathrm{D}$ & 0,85398 & 0,88496 & $-0,98$ & 0,329 \\
\hline \multirow[t]{2}{*}{ Edad del jefe del hogar } & A & 47,425 & 49,028 & $-1,40$ & 0,161 \\
\hline & $\mathrm{D}$ & 47,425 & 47,58 & $-0,11$ & 0,916 \\
\hline \multirow{2}{*}{$\begin{array}{l}\text { Edad del jefe del hogar } \\
\text { al cuadrado }\end{array}$} & $\mathrm{A}$ & 2490,5 & 2675,1 & $-1,53$ & 0,127 \\
\hline & $\mathrm{D}$ & 2490,5 & 2501,7 & $-0,07$ & 0,942 \\
\hline \multirow{2}{*}{$\begin{array}{l}\text { El jefe del hogar habla } \\
\text { alguna lengua indígena }\end{array}$} & A & 0,11062 & 0,22888 & $-4,12$ & $0,000 * * *$ \\
\hline & $\mathrm{D}$ & 0,11062 & 0,09292 & 0,62 & 0,535 \\
\hline \multirow{2}{*}{$\begin{array}{l}\text { Educación del jefe del } \\
\text { hogar (primaria } \\
\text { completa) }\end{array}$} & A & 0,24336 & 0,22363 & 0,68 & 0,499 \\
\hline & $\mathrm{D}$ & 0,24336 & 0,22566 & 0,42 & 0,658 \\
\hline \multirow{2}{*}{$\begin{array}{l}\text { Educación del jefe del } \\
\text { hogar (secundaria } \\
\text { completa) }\end{array}$} & $\mathrm{A}$ & 0,22566 & 0,1965 & 1,05 & 0,295 \\
\hline & $\mathrm{D}$ & 0,22566 & 0,23009 & $-0,11$ & 0,911 \\
\hline \multirow{2}{*}{$\begin{array}{l}\text { Educación del jefe del } \\
\text { hogar (preparatoria o } \\
\text { superior) }\end{array}$} & A & 0,15487 & 0,10678 & 2,20 & $0,028 * *$ \\
\hline & $\mathrm{D}$ & 0,15487 & 0,16372 & $-0,26$ & 0,798 \\
\hline \multirow{2}{*}{$\begin{array}{l}\text { Número de niños en el } \\
\text { hogar (<16 años) }\end{array}$} & $\mathrm{A}$ & 1,6062 & 1,3794 & 2,29 & $0,022 * *$ \\
\hline & D & 1,6062 & 1,677 & $-0,54$ & 0,586 \\
\hline \multirow{2}{*}{$\begin{array}{l}\text { Escolaridad más alta de } \\
\text { una mujer adulta en el } \\
\text { hogar }\end{array}$} & $\mathrm{A}$ & 9,3982 & 7,7168 & 5,72 & $0,000 * * *$ \\
\hline & $\mathrm{D}$ & 9,3982 & 9,3053 & 0,25 & 0,801 \\
\hline \multirow{2}{*}{$\begin{array}{l}\text { El hogar recibe } \\
\text { transferencias del } \\
\text { Gobierno }\end{array}$} & $\mathrm{A}$ & 0,53097 & 0,53479 & $-0,11$ & 0,913 \\
\hline & D & 0,53097 & 0,54425 & $-0,28$ & 0,778 \\
\hline \multirow{2}{*}{$\begin{array}{l}\text { Número de cuartos en } \\
\text { el hogar (sin contar } \\
\text { cocina y baño) }\end{array}$} & A & 3,1327 & 2,7195 & 4,18 & $0,000 * * *$ \\
\hline & $\mathrm{D}$ & 3,1327 & 3,1504 & $-0,13$ & 0,896 \\
\hline \multirow{2}{*}{$\begin{array}{l}\text { Proporción de } \\
\text { miembros del hogar } \\
\text { con buena salud }\end{array}$} & $\mathrm{A}$ & 0,52339 & 0,55456 & $-1,32$ & 0,186 \\
\hline & $\mathrm{D}$ & 0,52339 & 0,49089 & 1,04 & 0,299 \\
\hline \multirow{2}{*}{$\begin{array}{l}\text { El hogar tiene tierra } \\
\text { agrícola }\end{array}$} & A & 0,28761 & 0,30197 & $-0,45$ & 0,654 \\
\hline & $\mathrm{D}$ & 0,28761 & 0,27434 & 0,31 & 0,754 \\
\hline \multirow{2}{*}{$\begin{array}{l}\text { Superficie de la tierra } \\
\text { agrícola }\end{array}$} & $\mathrm{A}$ & 1,5015 & 1,0212 & 2,14 & $0,032 * * *$ \\
\hline & $\mathrm{D}$ & 1,5015 & 1,3208 & 0,47 & 0,642 \\
\hline \multirow{2}{*}{$\begin{array}{l}\text { Instituciones } \\
\text { financieras formales en } \\
\text { la localidad }\end{array}$} & A & 0,23894 & 0,15055 & 3,48 & 0,001 *** \\
\hline & $\mathrm{D}$ & 0,23894 & 0,19912 & 1,02 & 0,307 \\
\hline \multirow{2}{*}{$\begin{array}{l}\text { Prestamistas } \\
\text { informales en la }\end{array}$} & A & 0,31416 & 0,24902 & 2,14 & $0,032 * *$ \\
\hline & $\mathrm{D}$ & 0,31416 & 0,27876 & 0,82 & 0,411 \\
\hline
\end{tabular}

p-value: ***, ** * : significativos al $1 \%, 5 \%$ y $10 \%$, respectivamente.

Fuente: elaboración propia con base en Coneval (2013)

I A: antes del matching; D: después del matching

2 Nota: se está probando la igualdad de medias entre tratados y controles; la hipótesis nula establece que la media de la variable en los grupos de control y tratamiento es la misma (es decir, la variable está bien balanceada). De las i6 variables, 9 no están balanceadas antes del matching; sin embargo, después del matching, todas las variables están balanceadas. 
TABLA A.3

Prueba de balance general

\begin{tabular}{lcccc}
\hline \multicolumn{1}{c}{ Muestra } & $\begin{array}{c}\text { Media del } \\
\text { sesgo }\end{array}$ & $\begin{array}{c}\text { Pseudo- } \\
\mathbf{R}^{2}\end{array}$ & LR & Sesgo \\
\hline Antes del matching & 15,1 & 0,056 & $\begin{array}{c}85,59 \\
(0,000)\end{array}$ & 67,0 \\
\hline Después del matching & 4,3 & 0,008 & $5,27(0,994)$ & 21,6 \\
\hline
\end{tabular}

Fuente: elaboración propia con base en Coneval (2013)

Nota: la pseudo-R2 cae después del matching, lo que indica que las variables están bien balanceadas.

\section{Notas}

* Artículo de investigación

Licencia Creative Commons CC BY-4.० 